\title{
Assessment of three successive treatments of ranibizumab on neovascular macular degeneration by OCT angiography
}

\author{
JUMING ZHU ${ }^{1,2}$, QING PENG ${ }^{3}, \mathrm{TU} \mathrm{SU}^{3}$, MINLI WANG $^{3}$ and FANG WANG ${ }^{3}$ \\ ${ }^{1}$ Department of Ophthalmology, Nanjing Medical University, Nanjing, Jiangsu 210029; ${ }^{2}$ Department of Ophthalmology, \\ The Fourth Affiliated Hospital of Nantong University, Yancheng City No. 1 People's Hospital, Yancheng, \\ Jiangsu 224000; ${ }^{3}$ Department of Ophthalmology, Tenth People's Hospital, Shanghai 200072, P.R. China
}

Received October 9, 2019; Accepted September 18, 2020

DOI: $10.3892 /$ etm.2020.9491

\begin{abstract}
In the present study, the efficacy of three consecutive anti-VEGF treatments in patients with neovascular macular degeneration (nAMD) was assessed by optical coherence tomography angiography (OCT-A). A total of 23 eyes with nAMD were treated with intravitreal injections (IVIs) of ranibizumab once a month for three consecutive months. There were eight follow-up time-points: Prior to the initial IVI and on days 1, 3, 7, 14, 30, 60 and 90 after the first IVI. The follow-up examinations consisted of measurements of the best-corrected visual acuity (BCVA), the height of retinal pigmented epithelium detachment (RPED), the greatest linear dimension (GLD), choroidal neovascularization (CNV) flow area, whole retinal thickness and four-quadrant retinal thickness. The results indicated that, when compared with the baseline, the BCVA gradually improved after IVI and improved significantly on days 60 and $90(\mathrm{P}<0.05)$; the RPED and GLD improved after IVI, with a significant improvement on days 30, 60 and 90 (all, $\mathrm{P}<0.05$ ); the CNV flow area was gradually shortened in the early stage after IVI but rebounded on day 30 , while the difference was statistically significant on day $14(\mathrm{P}<0.05)$. The temporal retinal thickness was statistically significant on day $30(\mathrm{P}<0.05)$ and there were statistically significant differences in nasal and inferior retinal thickness on days 14 and 30 compared with prior to IVI (all, P<0.05). The BCVA was positively correlated with the RPED, GLD, CNV flow area and nasal retinal thickness (all, $\mathrm{P}<0.05)$. In conclusion, OCT-A may provide an effective reference to guide the evaluation, treatment and monitoring of nAMD. The present study was registered in the Chinese
\end{abstract}

Correspondence to: Professor Fang Wang, Department of Ophthalmology, Tenth People's Hospital, 301 Middle Yan Chang Road, Shanghai 200072, P.R. China

E-mail: fwang7527@163.com

Key words: neovascular macular degeneration, anti-vascular endothelial growth factor, optical coherence tomography angiography
Clinical Trial Registry (CChiTR; no. ChiCTR1900023607; http://www.chictr.org.cn/listbycreater.aspx).

\section{Introduction}

Age-related macular degeneration (AMD) is the primary cause of irreversible visual impairment in elderly individuals (1). Patients with AMD are generally $>50$ years of age and present with progressive degeneration in both eyes. AMD is roughly divided into two categories: Dry AMD and wet AMD (2). Wet AMD, also known as exudative AMD or neovascular AMD (nAMD), is the most severe form of visual impairment $(3,4)$. The pathological features of nAMD mainly include progressive atrophy of the retinal pigmented epithelium (RPE), Bruch's membrane and choroidal capillaries, resulting in the formation of choroidal neovascularization (CNV) $(5,6)$. The formation and development of $\mathrm{CNV}$ have a crucial role in the whole process of nAMD. Therefore, in recent years, anti-VEGF drugs to inhibit CNV have been used for the clinical treatment of nAMD.

Fundus fluorescein angiography (FFA) and indocyanine green angiography (ICGA) are thought to be the gold standard in terms of unambiguous diagnosis, classification, positioning and active monitoring of $\mathrm{nAMD}$, but the invasiveness of the examination-due to requiring intravenous dye-may rarely cause nausea, vomiting and severe allergic reactions. In the past 20 years, optical coherence tomography (OCT), particularly spectral domain OCT, has fundamentally changed the methods of diagnosis of macular disease, providing a non-invasive imaging method that is sensitive to the recognition of retinal and subretinal lesions. However, as both drusen and subretinal hemorrhage are characterized by high reflection, OCT has numerous limitations in differentiating between angiogenesis and dry AMD. OCT angiography (OCT-A) is one of the most significant advances in fundus imaging. It is able to display $3 \mathrm{D}$ retinal and choroidal vessels without any dye injection, so it is entirely non-invasive $(7,8)$. In the past three years, a large number of studies, both in clinical and experimental environments, have been conducted on the surface of the eye to evaluate the depth resolution of retinal and choroidal blood flow through the qualitative and quantitative analysis of OCT-A measurements $(9,10)$. In addition, it may be used to observe the activity or the resting state of CNV (11-13). 
In the present study, nAMD cases treated with ranibizumab were prospectively enrolled and the treatment outcomes were evaluated following OCT-A-based analyses.

\section{Materials and methods}

Patient selection and study design. The present study was a prospective analysis of patients undergoing individualized treatment for $n A M D$ recruited at the Department of Ophthalmology at Shanghai 10th People's Hospital (Shanghai, China) and was performed in compliance with the tenets of the Declaration of Helsinki. Approval was obtained from the Ethics Committee of Shanghai 10th People's Hospital (Shanghai, China; no. SHSY-IEC-4.0/19-41/01). All patients signed an informed consent form prior to inclusion. In this prospective study, 23 eyes of 18 patients with nAMD were examined. The patients first received three consecutive monthly injections of ranibizumab between April 2018 and November 2018.

Inclusion and exclusion criteria. All patients with nAMD underwent comprehensive ophthalmic examination, including best-corrected visual acuity (BCVA), slit lamp biomicroscopy, color fundus photography, fluorescein angiography, FFA/ICGA using a fundus camera (Spectralis; Heidelberg Engineering, Inc.) and OCT-A with an RTVue XR AngioVue Version 2017 (Optovue, Inc.). Furthermore, the diagnosis of nAMD in all patients was obtained from FFA/ICGA. To establish the presence of active CNV, patients must exhibit fluorescence leakage, as seen on FFA/ICGA, and hemorrhage, as seen on OCT-A, located either within or below the retina or below the RPE. The inclusion criteria for patients with nAMD in the present study were as follows: i) aged between 55 and 75 years; ii) nAMD with no previous treatment by methods including intravitreal injection (IVI) of drugs, photodynamic therapy and systemic or topical use of steroids. The exclusion criteria were a history of retinal diseases other than nAMD, glaucoma, uveitis, diabetes mellitus, rubeosis iridis, ocular infection, laser photocoagulation and intraocular surgery. Eyes that could not be imaged clearly by OCT-A were also excluded.

Study procedures. Patients with nAMD were scheduled to receive three doses of intravitreal anti-VEGF injection (ranibizumab; IVI of $0.5 \mathrm{mg}$ in $0.05 \mathrm{ml}$; Lucentis; Genentech, Inc.) monthly.

The patients with nAMD were examined prior to the first IVI and on days 1, 3, 7, 14, 30, 60 and 90 after the first IVI. The second injection was administered after the follow-up examination on day 30 and the third injection was given after the follow-up on day 60. Follow-up examination included BCVA, slit-lamp biomicroscopy, color fundus photography and OCT-A.

OCT-A imaging. OCT-A scans of a $6 \times 6 \mathrm{~mm}^{2}$ area centered on the fovea were acquired using an RT Vue-XR Avanti system (Optovue, Inc.) with AngioVue software (RTVue-XR version 2017.1.0.155; Optovue Inc.). This device uses a light source centered on a wavelength of $840 \mathrm{~nm}$ with a full-width at half-maximum bandwidth of $45 \mathrm{~nm}$ and an A-scan rate of 70,000 scans per second. At each location on the retina, the RTVue system captured two consecutive B-scans, each containing 304 A-scans. OCT-A evaluates the height of RPE detachment (RPED), the greatest linear dimension (GLD), CNV flow area, whole retinal thickness and four-quadrant retinal thickness (Fig. 1).

Fig. 1 displays representative measurements of the macula in an eye with nAMD by using OCT-A on a 6x6 mm scan. The inner ring and outer ring were subdivided into four sectors (superior, nasal, inferior and temporal). In Fig. 1A and B, the superficial and deep retinal thicknesses were measured in the fovea $(1 \mathrm{~mm})$, inner ring $(3 \mathrm{~mm})$ and outer ring $(6 \mathrm{~mm})$ following the Early Treatment of Diabetic Retinopathy Study grid. The OCT scan (horizontal B-scan) provides segmentation of individual retinal layers, and on this diagram, the GLD and RPED may be measured. Furthermore, the CNV flow area was measured by AngioVue software (Fig. 1D and E). The OCT-A images were graded by two observers.

Statistical analysis. The parameters were presented and analyzed using typical methods for descriptive statistics. The mean and standard deviation were calculated for quantitative parameters. Repeated-measures ANOVA with Bonferroni adjustment was performed to make comparisons between each follow-up time-point with the baseline. Pearson's correlation test was used to study the correlation between BCVA and quantified OCT-A measurements in patients with nAMD at the baseline prior to the first IVI. $\mathrm{P}<0.05$ was considered to indicate statistical significance. All statistical analyses were performed using the IBM SPSS Statistics (version 21.0; IBM Corp.).

\section{Results}

Patient characteristics. A total of 23 eyes from 18 patients (12 males and 6 females; age, $65.61 \pm 4.88$ years) with CNV were included in the present study and all eyes had type II CNV. The baseline and follow-up results of the 23 eyes with nAMD are presented in Table I.

\section{OCT-A results in $n A M D$ patients}

Changes in BCVA. The BCVA gradually improved after IVI and improved significantly on days 60 and 90 in comparison to the baseline (each, $\mathrm{P}<0.05$; Table I).

Changes in CNV. In Table I, the RPED also improved after IVI with a significant improvement on days 30 and 60 relative to the baseline (each, $\mathrm{P}<0.05$ ), but it was slightly increased on day 90 compared with the baseline $(\mathrm{P}<0.05)$. The follow-up observations also indicated that the length of the GLD was gradually shortened after IVI and the difference was statistically significant from day 30 to day 90 (all, $\mathrm{P}<0.05$ ). In addition, the flow area of the CNV exhibited a trend of improvement after IVI, which also became slightly elevated again on day 90; however, the difference was statistically significant only on day 14 after IVI $(\mathrm{P}<0.05)$.

Fig. 2 presents examination images of a 66-year-old male with nAMD. Fundus color photographs revealed a yellow-white disc-shaped lesion in the macula, surrounded by small pieces indicative of bleeding. The bleeding was gradually absorbed after IVI and there was no obvious bleeding in the macula at 

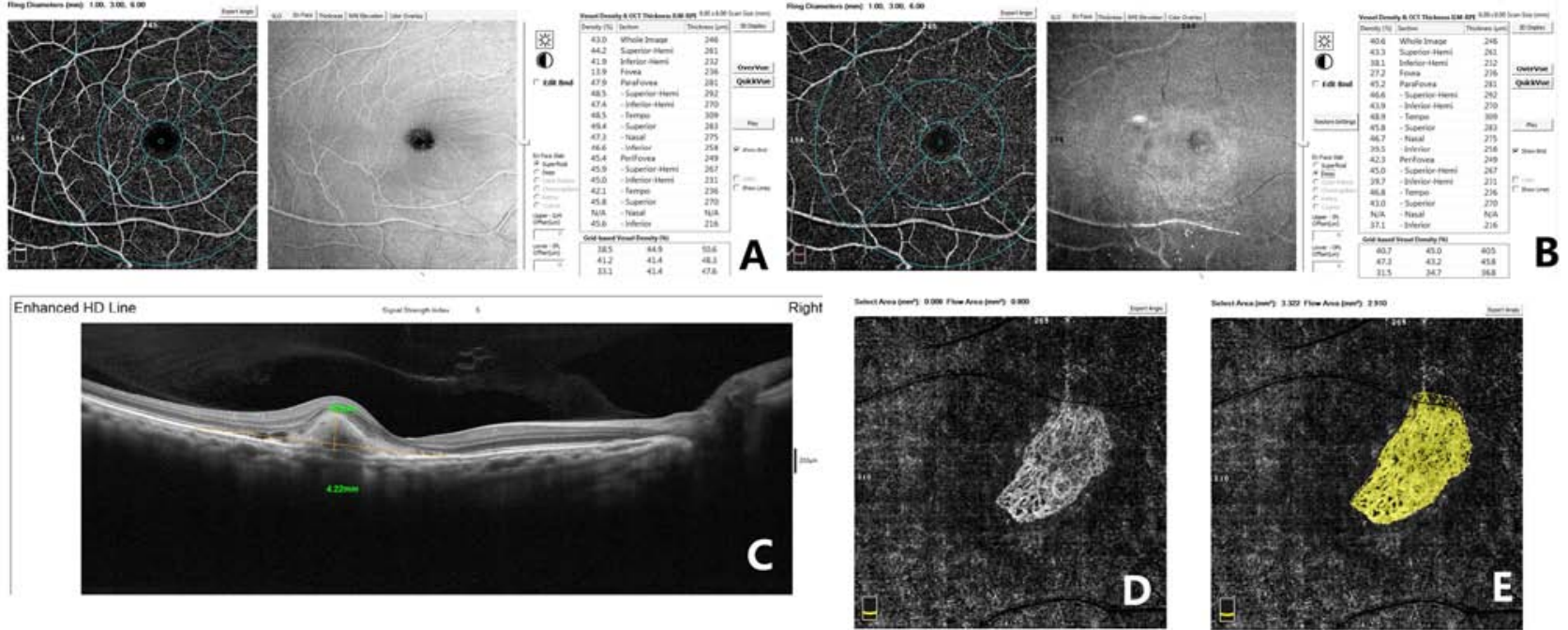

Figure 1. Representative measurement of the macula in an eye with neovascular macular degeneration with OCT-angiography on a $6 \times 6 \mathrm{~mm} s c a n$. (A) Superficial and (B) deep retinal thicknesses were measured in the fovea $(1 \mathrm{~mm})$, inner ring $(3 \mathrm{~mm})$ and outer ring $(6 \mathrm{~mm})$ following the Early Treatment of Diabetic Retinopathy Study grid. The inner ring and outer ring are subdivided into four sectors (superior, nasal, inferior and temporal). (C) OCT scan (horizontal B-scan) through the macula indicates segmentation of individual retinal layers; the greatest linear dimension and retinal pigmented epithelium detachment can be measured on this diagram. (D) Choroidal neovascularization is presented via OCT-angiography, and (E) choroidal neovascularization flow area is measured by AngioVue software. OCT, optical coherence tomography; RPE, retinal pigmented epithelium; ILM, inner limiting membrane.
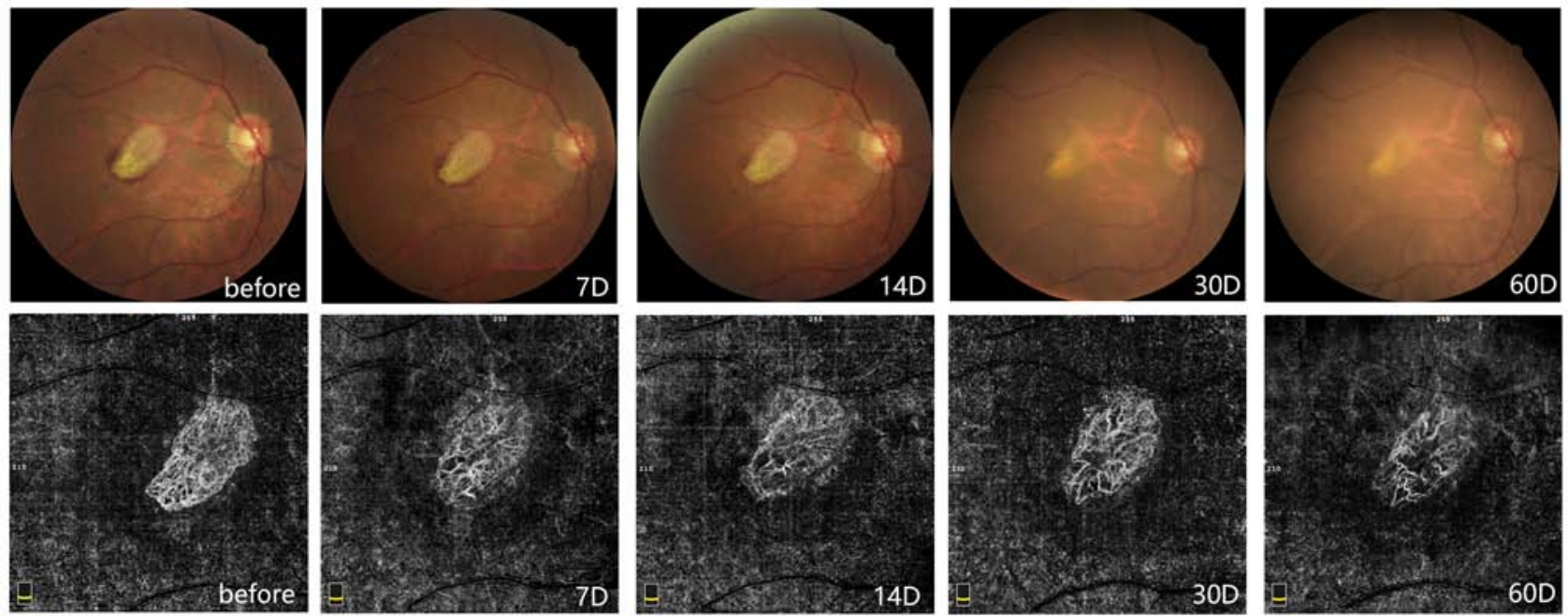

Figure 2. Fundus color photographs and CNV measured by OCT-A at five follow-up time-points. The fundus color photograph at the baseline prior to IVI revealed a yellow-white disc-shaped lesion in the macula, surrounded by small pieces indicative of bleeding. The bleeding was gradually absorbed after IVI and there was no obvious bleeding in the macula at day 60. OCT-A suggested that the CNV had dense vascular branches prior to IVI, with secondary branches and an abnormal vascular arch. After treatment, the CNV exhibited a gradually reduced blood flow density and vascular branches became more sparse. CNV, choroidal neovascularization; IVI, intravitreal injection; OCT-A, optical coherence tomography angiography; D, days.

day 60. OCT-A suggested that the CNV had dense vascular branches prior to IVI, with secondary branches and an abnormal vascular arch. With the gradual improvement of the disease after treatment, the CNV presented with a gradually reduced blood flow density, the vascular branches became more sparse and the surrounding vascular arch was interrupted. These abnormal new blood vessels gradually became thick, straight and stiff in appearance and lacked capillaries.

Changes in retinal thickness. The whole retinal thickness and the retinal thickness in the four quadrants exhibited a slow thinning on day 30 , followed by a slight thickening, with the thickness stabilizing on days 60-90. At the 7 follow-up time-points, there was no statistically significant difference in the whole retinal thickness and superior retinal thickness from that at the baseline. However, the temporal retinal thickness was significantly different on day $30(\mathrm{P}<0.05)$, and there were statistically significant differences in nasal retinal thickness and inferior retinal thickness on day 14 and day 30 compared with the baseline (all, $\mathrm{P}<0.05$; Table I).

Correlations between BCVA and the quantified OCT-A measurements. Finally, the correlations between the quantified OCT-A measurements and BCVA were 


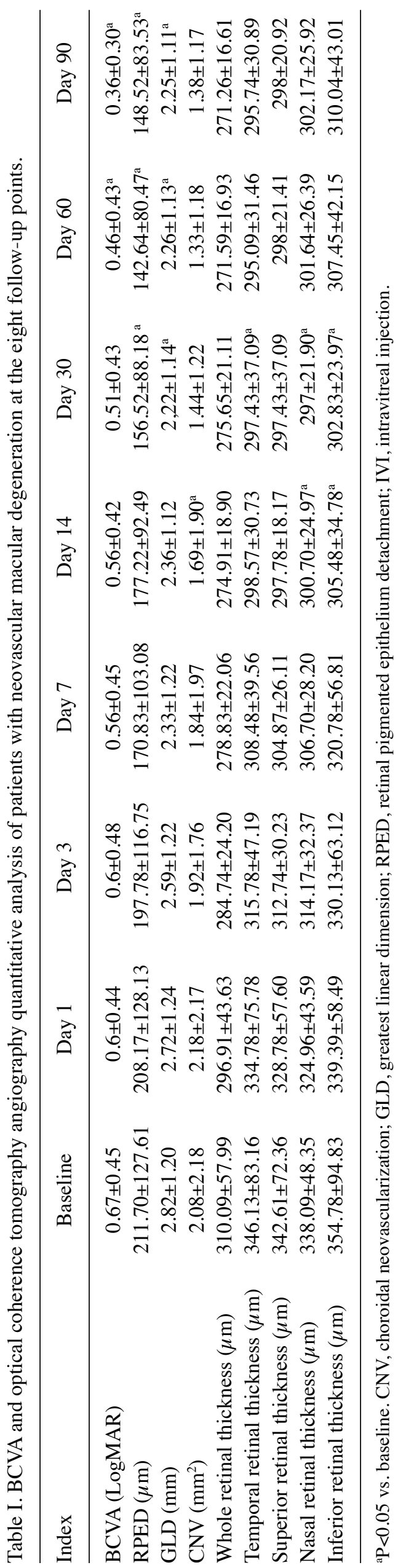

Table II. Correlations of best-corrected visual acuity with quantified optical coherence tomography angiography measurements in patients with neovascular macular degeneration prior to the first intravitreal injection.

Variable

Correlation parameters

RPED

$\mathrm{r}=0.26, \mathrm{P}=0.001$

GLD

$\mathrm{r}=0.26, \mathrm{P}=0.001$

$\mathrm{CNV}$

$\mathrm{r}=0.24, \mathrm{P}=0.002$

Whole retinal thickness

$\mathrm{r}=-0.07, \mathrm{P}=0.382$

Temporal retinal thickness

$\mathrm{r}=0.16, \mathrm{P}=0.054$

Superior retinal thickness

$\mathrm{r}=0.02, \mathrm{P}=0.831$

Nasal retinal thickness

$\mathrm{r}=-0.18, \mathrm{P}=0.021$

Inferior retinal thickness

$\mathrm{r}=0.03, \mathrm{P}=0.683$

r, Pearson correlation coefficient; $\mathrm{CNV}$, choroidal neovascularization; GLD, greatest linear dimension; RPED, retinal pigmented epithelium detachment.

analyzed prior to the first time of IVI by Pearson correlation tests (Table II). The results suggested that the BCVA was positively correlated with RPED ( $\mathrm{r}=0.26, \mathrm{P}=0.001)$, GLD ( $\mathrm{r}=0.26$, $\mathrm{P}=0.001), \mathrm{CNV}$ flow area $(\mathrm{r}=0.24, \mathrm{P}=0.002)$ and nasal retinal thickness $(\mathrm{r}=-0.18, \mathrm{P}=0.021)$.

\section{Discussion}

In the clinic, injection of anti-VEGF is mainly used to treat nAMD, which is able to effectively reduce the size of the CNV and the severity of vascular leakage. Fauser et al (14) indicated that both BCVA values of patients with nAMD were significantly improved and the expression level of VEGF was significantly decreased after patients were injected with anti-VEGF. However, certain studies indicated that anti-VEGF did not significantly improve the BCVA $(15,16)$. In the present study, the BCVA was gradually improved after treatment and the improvement on days 60 and 90 was statistically significant. Furthermore, the three indicators of RPED, GLD and CNV flow area, were determined in the present study, which exhibited a tendency of improvement in the early stage. The reason for this may be that the patients were treated with ranibizumab for the first time and were sensitive to the drug, and thus, the visual acuity was significantly improved. The present results also suggested that the three indicators were positively correlated with the BCVA, suggesting that these indicators are able to directly reflect the disease. The pathophysiological mechanism of nAMD is complex and known to be associated with oxidative damage $(17,18)$, immune response, aging, metabolic disorders and chronic inflammatory reactions $(1,19,20)$. Therefore, anti-VEGF alone cannot effectively control the development of nAMD for long periods of time and may require to be combined with other therapies at later stages, such as anti-inflammatory therapy.

In 2011, 1-year results of the Comparison of AMD Treatments Trials (CATT) (21) supported that both ranibizumab and bevacizumab reduced retinal and subretinal fluid, but ranibizumab eliminated fluid more. In addition, there were more serious adverse events in patients treated with 
bevacizumab. Therefore, ranibizumab is widely used for anti-VEGF therapy in patients with nAMD. However, in 2020, one CATT (22) suggested that ranibizumab and bevacizumab had similar effects on visual acuity over a 2-year period, and there were no differences between drugs in rates of death or arteriothrombotic events. Recently, another study indicated a similar systemic safety profile for bevacizumab and ranibizumab as intravitreal pharmacotherapies for common retinal conditions in routine clinical practice (23). In addition, bevacizumab is available at low cost and it may have a greater potential for clinical use. Regrettably, a large number of patients experienced ocular adverse events in Shanghai in September 2010 after treatment with bevacizumab (Avastin; Genentech) (24,25). Therefore, the application of bevacizumab in China is limited by policy implications (26).

The advantage of the present study is that changes in patients with nAMD after anti-VEGF injections were closely observed, but the disadvantages are that the observation time was short, the number of cases was small and changes in the condition of recurrent refractory nAMD were not observed. Therefore, further studies with an increased number of patients will be performed in the future to avoid the inclusion of two eyes from the same patient, thereby making the results more credible.

In conclusion, nAMD is a severe, blinding eye disease and its incidence is increasing year by year, but the available clinical treatments have no significant long-term effect, so it is urgent to develop novel drugs and methods that are efficient alongside these current treatments. OCT-A, a non-invasive imaging technique that allows for the qualitative and quantitative analysis of macular lesions, may provide an effective biomarker and guide the evaluation, treatment and monitoring of patients with nAMD.

\section{Acknowledgements}

Not applicable.

\section{Funding}

This study was funded by the National Natural Science Foundation of China (grant no. 81770939).

\section{Availability of data and materials}

The datasets used and/or analyzed during the current study available from the corresponding author on reasonable request.

\section{Authors' contributions}

JZ, QP and FW made substantial contributions to conception and design. JZ, QP and TS acquired the data. JZ and MW analyzed and interpreted the data. JZ and QP involved in drafting the manuscript. FW gave final approval for the version to be published. All authors read and approved the final manuscript.

\section{Ethics approval and consent to participate}

All procedures performed in studies involving human participants were in accordance with the ethical standards of the Ethics Committee of Shanghai 10th People's Hospital (Shanghai, China) and with the Declaration of Helsinki from 1964 and its later amendments or comparable ethical standards. Approval was obtained from the Ethics Committee of Shanghai 10th People's Hospital (Shanghai, China; no. SHSY-IEC-4.0/19-41/01). Prior written informed consent was obtained from all participants included in the study.

\section{Patient consent for publication}

Not applicable.

\section{Competing interests}

The authors declare that they have no competing interests.

\section{References}

1. Ambati J, Ambati BK, Yoo SH, Ianchulev S and Adamis AP: Age-related macular degeneration: Etiology, pathogenesis, and therapeutic strategies. Surv Ophthalmol 48: 257-293, 2003.

2. Anderson DH, Mullins RF, Hageman GS and Johnson LV: A role for local inflammation in the formation of drusen in the aging eye. Am J Ophthalmol 134: 411-431, 2002.

3. Jager RD, Mieler WF and Miller JW: Age-related macular degeneration. N Engl J Med 358: 2606-2617, 2008.

4. Ardeljan C, Ardeljan D, Abu-Asab M and Chan CC: Inflammation and cell death in age-related macular degeneration: An immunopathological and ultrastructural model. J Clin Med 3: 1542-1560, 2014.

5. Cho Y, Cao X, Shen D, Tuo J, Parver LM, Rickles FR and Chan CC: Evidence for enhanced tissue factor expression in age-related macular degeneration. Lab Investig 91: 519-526, 2011.

6. MacHalińska A, Kłos P, Safranow K, Dziedziejko V, Rudnicki M, Paczkowska E, Karczewicz D and Machaliński B: Neural stem/progenitor cells circulating in peripheral blood of patients with neovascular form of AMD: A novel view on pathophysiology. Graefes Arch Clin Exp Ophthalmol 249: 1785-1794, 2011.

7. Faridi A, Jia Y, Gao SS, Huang D and Bhavsar KV: Sensitivity and Speci fi city of OCT angiography to detect choroidal neovascularization. Ophthalmol Retin 1: 294-303, 2017.

8. Schneider EW and Fowler SC: Optical coherence tomography angiography in the management of age-related macular degeneration. Curr Opin Ophthalmol 29: 217-225, 2018.

9. Lupidi M, Cerquaglia A, Chhablani J, Fiore T, Singh SR, Cardillo Piccolino F, Corbucci R, Coscas F, Coscas G and Cagini C: Optical coherence tomography angiography in age-related macular degeneration: The game changer. Eur J Ophthalmol 28: 349-357, 2018.

10. Kaszubski PA, Ben Ami T, Saade C, Nabati C, Kumar V, Santos AR, Silva R, Cachulo ML, Cunha-Vaz JG and Smith RT: Changes in reticular pseudodrusen area in eyes that progressed from early to late age-related macular degeneration. Int Ophthalmol 38: 503-511, 2018.

11. Aggarwal K, Agarwal A, Sharma A, Sharma K and Gupta V; OCTA Study Group: Detection of type 1 choroidal neovascular membranes using optical coherence tomography angiography in tubercular posterior uveitis. Retina 39: 1595-1606, 2018.

12. Nesper PL, Soetikno BT, Treister AD and Fawzi AA: Volume-rendered projection-resolved OCT angiography: $3 \mathrm{D}$ lesion complexity is associated with therapy response in wet age-related macular degeneration. Investig Ophthalmol Vis Sci 59: 1944-1952, 2018.

13. Pilotto E, Frizziero L, Daniele AR, Convento E, Longhin E, Guidolin F, Parrozzani R, Cavarzeran F and Midena E: Early OCT angiography changes of type $1 \mathrm{CNV}$ in exudative AMD treated with anti-VEGF. Br J Ophthalmol 103: 67-71, 2019.

14. Fauser S, Schwabecker V and Muether PS: Suppression of intraocular vascular endothelial growth factor during aflibercept treatment of age-related macular degeneration. Am J Ophthalmol 158: 532-536, 2014. 
15. Heier JS, Brown DM, Chong V, Korobelnik JF, Kaiser PK, Nguyen QD, Kirchhof B, Ho A, Ogura Y, Yancopoulos GD, et al: Intravitreal aflibercept (VEGF trap-eye) in wet age-related macular degeneration. Ophthalmology 119: 2537-2548, 2012.

16. Liu F, Ding X, Yang Y, Li J, Tang M, Yuan M, Hu A, Zhan Z $\mathrm{Li} \mathrm{Z}$ and $\mathrm{Lu} \mathrm{L}$ : Aqueous humor cytokine profiling in patients with wet AMD. Mol Vis 22: 352-361, 2016.

17. Nowak JZ: Age-related macular degeneration (AMD): Pathogenesis and therapy. Pharmacol Rep 58: 353-363, 2006.

18. Holly field JG, Bonilha VL, Rayborn ME, Yang X, Shadrach KG, Lu L, Ufret RL, Salomon RG and Perez VL: Oxidative damage-induced inflammation initiates age-related macular degeneration. Nat Med 14: 194-198, 2008.

19. Chen $\mathrm{M}$ and $\mathrm{Xu} \mathrm{H}$ : Parainflammation, chronic inflammation, and age-related macular degeneration. J Leukoc Biol 98: 713-725, 2015.

20. Xu H, Chen M and Forrester JV: Para-inflammation in the aging retina. Prog Retin Eye Res 28: 348-368, 2009.

21. CATT Research Group; Martin DF, Maguire MG, Ying GS, Grunwald JE, Fine SL and Jaffe GJ: Ranibizumab and bevacizumab for neovascular age-related macular degeneration. N Engl J Med 364: 1897-1908, 2011.
22. Comparison of Age-related Macular Degeneration Treatments Trials (CATT) Research Group; Writing Committee; Martin DF, Maguire MG, Fine SL, Ying GS, Jaffe GJ, Grunwald JE, Toth C, Redford M and Ferris FL III: Ranibizumab and bevacizumab for treatment of neovascular age-related macular degeneration. Ophthalmology 127 (Suppl): S135-S145, 2020.

23. Shah ND and Barkmeier AJ: Risk of systemic adverse events following intravitreal bevacizumab, ranibizumab, and aflibercept in routine clinical practice. Ophthalmology: Aug 8, 20202020 (Epub ahead of print).

24. Sun $X, X u X$ and Zhang X: Counterfeit bevacizumab and endophthalmitis. N Engl J Med 365: 378-379, 2011.

25. Wang F, Yu S, Liu K, Chen FE, Song Z, Zhang X, Xu X and Sun X: Acute intraocular inflammation caused by endotoxin after intravitreal injection of counterfeit bevacizumab in Shanghai China. Ophthalmology 120: 355-361, 2013.

26. Huang HB, Pan Y and Liu T: Shanghai eye treatment outbreak: Bevacizumab therapy for AMD in China. Clin Exp Optom 96: 106-108, 2013. 